\title{
Diagnóstico ecológico para la conservación de Jatropha spp. (Euphorbiaceae) y sus hábitats, en la Reserva Tehuacán-Cuicatlán, México
}

\author{
Nancy Hernández-Nicolás ${ }^{1}$, Leobigildo Córdova-Téllez ${ }^{1}$, Angélica Romero-Manzanares ${ }^{1 *}$, Jaime \\ Jiménez-Ramírez ${ }^{2}$, Ricardo Lobato-Ortiz ${ }^{1} \&$ Jesús Cuevas-Sánchez $^{3}$ \\ 1. Colegio de Postgraduados, Programas de Genética y Botánica. Km. 36.5 Carr. México-Texcoco. 56 230, Montecillo, \\ Texcoco, Estado de México; jazzz_hdz@hotmail.com, lcordova@colpos.mx, dahly@colpos.mx, rlobato@colpos.mx \\ 2. Universidad Nacional Autónoma de México, Ciudad Universitaria, circuito exterior. Col. Copilco el Bajo, Del. \\ Coyoacán. AP 04 510. México, DF; jjimenez_ramirez@yahoo.com \\ 3. Universidad Autónoma Chapingo. Chapingo, Estado de México. 56 230. Km 38.5 Carretera México-Texcoco; \\ jaxayacatl@gmail.com \\ * Dirección de correspondencia.
}

Recibido 12-III-2014. Corregido 30-VII-2014. Aceptado 27-VIII-2014.

\begin{abstract}
Diagnosis for the ecological conservation of Jatropha spp. (Euphorbiaceae) and their habitats in the Tehuacan-Cuicatlan Reserve, Mexico. Jatropha spp. is an important phytogenetic resource used as food, medicine, and biofuel. In this study, we verified the taxonomic identity of Jatropha species for The Biosphere Reserve Tehuacan-Cuicatlan, and the Ecological Land Units (ELU) occupied by them. We assessed the conservation status of their habitats, and the vulnerability of Jatropha spp. populations. A total of 15 sampling sites were selected in the Reserve. The taxonomic work was based on specimens, original descriptions and type material from herbaria and those available on-line. ELUs were classified using biophysical variables, and gvSIG software. Ecological attributes were determined using a quantitative analysis by the point-centered quarter method; disturbance was estimated through site indicators, and the conservation status of the Jatropha populations was assessed using the Method for Evaluation of the Risk of Extinction of Plants in Mexico (MER). Jatropha frequently dominated the physiognomy of plant communities. The current distribution of Jatropha species in the Reserve was mainly determined by elevation, temperature, and precipitation variables. The confirmed species were Jatropha ciliata Sessé ex Cerv., Jatropha neopauciflora Pax, Jatropha oaxacana J. Jiménez Ram. \& R. Torres, Jatropha rufescens Brandegee, and Jatropha rzedowskii J. Jiménez Ram., which are distributed in four of the six defined ELU. J. neopauciflora and J. rzedowskii were the most widespread species; this last species concur in four, J. oaxacana in two, while $J$. rufescens and J. ciliata in one ELU, being the most restricted. The richness of the genera in the associated communities ranged from 16 to 42 . The maximum and minimum Importance Value Indexes were observed in San Nicolas Tepoxtitlan for J. neopauciflora (53.75\%) and J. rzedowskii $(1.50 \%)$. The disturbance index varied from 0.22 to 0.82 , with an average of 0.51 , where the livestock variable had a high contribution. Considering the risk categories of MER, we concluded that J. oaxacana requires special protection, and J. ciliata and J. rufescens were species under extinction risk. The conservation status of the plant communities inhabited by populations of Jatropha spp. was moderate, taking into account the rate of disturbance. The main factors affecting the habitats of Jatropha were livestock and human activities; the floristic composition of the surrounding plant communities has low richness. These results support the urgent need of conservation strategies to avoid the loss of important wild growing species of Jatropha populations. Rev. Biol. Trop. 63 (1): 23-33. Epub 2015 March 01.
\end{abstract}

Key words: Jatropha species, ecological land units, Tropical Deciduous Forest, disturbance, vulnerability, Mexico.

El aumento de las poblaciones humanas y la intensificación del uso del suelo a través del cultivo, pastoreo y desarrollo urbano, han dado lugar a la pérdida y fragmentación de los hábitats naturales. Las consecuencias de estos hechos afectan la diversidad genética de las especies, la pérdida de la biodiversidad y el aumento en la vulnerabilidad, hasta el riesgo 
de extinción (Wilson, \& Provan, 2003). Conservar el acervo genético contribuiría a evitar la pérdida de recursos que, en un futuro, eventualmente resolverían problemas tan significativos como la falta de alimento, de medicinas y de otras necesidades.

Jatropha (Euphorbiaceae) es un recurso fitogenético que potencialmente cubriría las necesidades mencionadas; está representado por 186 especies, es nativo de América Central y México, y se distribuye también en África, Asia y la Polinesia (Dehgan, 2012). México es rico en especies nativas de Jatropha, alberga a 45 taxones de este género $(20 \%$ del total mundial), de los cuales 35 (77\%) son endémicos (Martínez et al., 2002). En cuanto a la distribución geográfica en México de las especies de Jatropha, Córdova (com. pers., 2013) propone ocho regiones importantes con alta riqueza genética de especies del género, y menciona, que una de las regiones que más contribuye a la biodiversidad de Jatropha es el Valle de Tehuacán-Cuicatlán, el cual contiene 12 especies, riqueza que a diferencia de la opinión de Jiménez (com. pers., 2013), se reduce a seis taxones.

Sobre la distribución ecológica de las especies de Jatropha en la Reserva TehuacánCuicatlán, solo se cita a $J$. curcas L. en huertos, a J. dioica Sessé en el Bosque Tropical Caducifolio (Selva Baja Caducifolia) o en la tetechera (comunidad dominada por cactáceas columnares de Neobuxbaumia tetetzo (J.M. Coult.) Backeb) y, a J. neopauciflora Pax, en los huertos y las barrancas (Dávila-Aranda, \& Lira-Saade, 2002). Para las demás especies de Jatropha se desconocen tanto las características del hábitat que ocupan, como el estado actual de conservación de las comunidades donde habitan.

El área de la Reserva Tehuacán-Cuicatlán tiene una larga historia de ocupación humana (Byers, 1967); en este espacio, la población rural realiza actividades de ganadería extensiva, agricultura y extracción de productos de recolección, lo que afecta a Jatropha spp., por la transformación del paisaje y la fragmentación de los hábitats, causas que amenazan la persistencia de algunas comunidades únicas y principalmente, a las especies endémicas (Anderson, 1990).

Para llevar a cabo esta investigación se considera que el concepto base en estudios ecológico-etnobotánicos y para la conservación, es la Unidad Ambiental (UA o ELU por sus siglas en inglés: Ecological Land Units), definida como zona homogénea de un ecosistema natural, cuya delimitación y estructuración considera parámetros físicos y la biodiversidad, a través de las comunidades vegetales y sus especies, la agrodiversidad y la diversidad de aprovechamientos (Dávila-Aranda, \& LiraSaade, 2002; Paredes-Flores, Lira, \& Dávila, 2007; Zimmerman, \& Runkle, 2010).

Los objetivos propuestos fueron: precisar la identidad de las especies de Jatropha de la Reserva Tehuacán-Cuicatlán, diferenciar las unidades ambientales en las que habitan, valorar el estado de conservación de los hábitats y, calificar la vulnerabilidad de las poblaciones de Jatropha, para las especies reconocidas.

\section{MATERIALES Y MÉTODOS}

Área de estudio: La región de estudio se acota a la Reserva de la Biosfera Tehuacán-Cuicatlán, que se encuentra en el sureste del estado de Puebla y noreste de Oaxaca (17 32'24' $18^{\circ} 52^{\prime} 55^{\prime \prime} \mathrm{N}-96^{\circ} 59^{\prime} 24^{\prime \prime}$ - 9748'43” W), altitud de 200 a $3500 \mathrm{msnm}$, donde predomina un ambiente semiárido, con presencia de Matorrales Xérofilos y Selva Baja Caducifolia, Bosque Espinoso, Bosque de Encino, Bosque de PinoEncino y Pastizal (DOF, 1998). De acuerdo con la clasificación de Köppen, modificada por García (1990), el clima es cálido semidesértico $\mathrm{BS}_{0} \mathrm{hw}$ " $(\mathrm{w})(\mathrm{e})(\mathrm{g})$, con medias anuales de temperatura de $21^{\circ} \mathrm{C}$ y precipitación $380 \mathrm{~mm}$.

Muestreo: Para decidir sobre las localidades y número de sitios de muestreo dentro de la Reserva, primero se procedió a revisar los ejemplares de Jatropha localizados en diversos repositorios (Herbario Nacional MEXUUNAM; Herbario-Hortorio CHAPA-COLPOS; Herbario de Plantas Útiles XOLO-UACH; Herbario de Ciencias Forestales CHP-UACH; 
Herbario de Preparatoria Agrícola UACH; Herbario del Instituto de Ecología A. C., México (IE-Bajío)-IEB; Red Mundial sobre Biodiversidad REMIB-CONABIO). Se obtuvieron 67 registros del género Jatropha en 31 localidades. Con base en el número de localidades obtenidas de estos registros se calculó el número de muestreos requeridos para la investigación, de acuerdo con la siguiente ecuación (Murray, 1988):

$$
n=\frac{N S^{2}}{\frac{B_{\mu}^{2}}{t^{2}}(N-1)+S^{2}}
$$

Donde:

$n=$ Tamaño de muestra; $N=$ Tamaño de la población (31 localidades); $S^{2}=$ Varianza ponderada de la población (varianza máxima de 0.25$) ; B_{\mu}^{2}$ $=$ Precisión expresada en centésimas $(0.20$, lo que equivale a $20 \%$ ); $t=$ Valor de la distribución "z" para una confiabilidad dada (para poblaciones menores a 100 registros la distribución " $t "=2.0860$ ). Con un error de precisión del $20 \%$ se seleccionaron 15 localidades para realizar el muestreo; los criterios de selección fueron dos: accesibilidad, por distancia de recorrido más presencia de caminos y, el número de especies verificadas taxonómicamente.

Identidad de especies: Las especies fueron colectadas y determinadas basándose en las descripciones originales de Jatropha rzedowskii J. Jiménez Ram. (Jiménez, 1985); Jatropha oaxacana J. Jiménez Ram. \& R. Torres (Jiménez \& Torres, 1994); Jatropha rufescens Brandegee (Brandegee, 1910); Jatropha ciliata Sessé (Cervantes, 1794); Jatropha neopauciflora Pax (Pax, 1910). Además, se hizo uso del material tipo de herbarios y se trabajó con los holotipos e isotipos disponibles en internet (Tropicos, 2014; Jstor, 2012).

Unidades ambientales (UA): Las unidades ambientales se delimitaron con un análisis multicriterio (Barredo, 1996) y la herramienta Intersect del software de Sistemas de Información Geográfica gvSIG; con respecto a las variables, se combinaron en formato vector la temperatura mínima-máxima, elevación $(\mathrm{msnm})$, precipitación media anual, amplitud climática de humedad, amplitud climática de temperatura, régimen de humedad, tipo de suelo, sustrato, relieve, uso de suelo y vegetación (CONABIO, 2013); las variables ponderadas fueron temperatura, precipitación y elevación.

Atributos ecológicos de las comunidades vegetales: La vegetación fue trabajada con un método sin área, el cuadrante centrado en un punto, cuya metodología y modificaciones prácticas las detalla Mitchell (2007). En cada sitio se tendieron 20 líneas de $100 \mathrm{~m}$ al azar, con una marca cada $10 \mathrm{~m}$ para indicar el origen de los cuadrantes; por cada línea se seleccionaron cinco puntos al azar. En cada cuadrante se midió la distancia del árbol o arbusto más cercano identificándolo a género, y en el caso de Jatropha, hasta especie; a cada individuo registrado se le midió el diámetro del dosel en dos dimensiones para calcular la cobertura. Se estimaron los atributos ecológicos de las comunidades vegetales y el índice de valor de importancia (IVI) relativo, tanto por especie de Jatropha como para cualquier otro género en la comunidad bajo estudio. Con el listado de los taxones registrados se realizaron curvas de Rango-Abundancia por sitio; la secuencia de ordenación derivó de la relación $\log _{10}$ de la proporción (pi) de individuos (Feinsinger, 2001) de cada género y de las especies de Jatropha; éstas curvas permitieron describir en orden descendente, la estructura de la comunidad, basada en la riqueza, abundancia y equidad de las especies o géneros.

Disturbio: Por cada sitio de muestreo se tomó una de las 20 líneas de 100m utilizadas previamente y se seleccionaron al azar cinco de los cuadrantes trabajados, a los que se aplicó el método de Martorell y Peters (2005), para el cálculo de la intensidad de disturbio. Para esto se emplearon 15 indicadores agrupados en tres rubros: ganadería, actividades humanas y degradación de la tierra. Se calculó el índice de disturbio (ID) con la frecuencia relativa de cada 
uno de los indicadores y la suma total de estos; el índice se acota entre 0 y 1 (nulo a máximo disturbio). Los datos se estandarizaron y resumieron a través de un análisis de componentes principales (ACP) utilizando el programa SAS versión 9.0.

Estado de conservación de la poblaciones de Jatropha: Con los datos generados en campo, la información de artículos científicos y registros de herbario, se aplicó el protocolo conocido como "Método de Evaluación del Riesgo de Extinción de Plantas en México (MER)" de la NOM-059-SEMARNAT (DOF, 2010), que asigna un estatus de riesgo a cualquier especie silvestre a través de la valoración de los criterios: (A) Características de la distribución geográfica; (B) Características del hábitat; (C) Vulnerabilidad biológica intrínseca; y (D) Impacto de la actividad humana. Los cuatro criterios tienen un valor máximo 1 . Las categorías derivadas de este método de evaluación de riesgo fueron: En Peligro de Extinción ( $\mathrm{P}=$ mayor o igual a 2 puntos), Amenazada $(A=$ mayor que 1.7 y menor que 2), Sujetas a
Protección Especial ( $\mathrm{Pr}=$ mayor o igual que 1.5 y menor que 1.7).

\section{RESULTADOS}

Especies y unidades ambientales: Las especies de Jatropha registradas y cotejadas para la región de estudio fueron: $J$. neopauciflora, J. ciliata Sessé ex Cerv., J. oaxacana J. Jiménez Ram. \& R. Torres, J. rufescens Brandegee y J. rzedowskii J. Jiménez Ram., que se distribuyeron únicamente en cuatro de las seis unidades ambientales diferenciadas (Fig. 1).

Las variables que definieron las unidades ambientales fueron temperatura, precipitación y elevación, y en aquellas en las que se distribuyeron las especies de Jatropha, se caracterizaron por presentar temperatura mínima superior a $0^{\circ} \mathrm{C}$, precipitaciones por arriba de $200 \mathrm{~mm}$ y elevaciones inferiores a $2500 \mathrm{~m}$. En la UA2 se distribuyó $J$. oaxacana, $J$. neopauciflora y $J$. rzedowskii; en la UA3 J. rufescens, J. ciliata, J. oaxacana, J. neopauciflora, J. rzedowskii; en la UA4, J. rzedowskii, J. neopauciflora; y en la UA5, J. rzedowskii y J. neopauciflora.

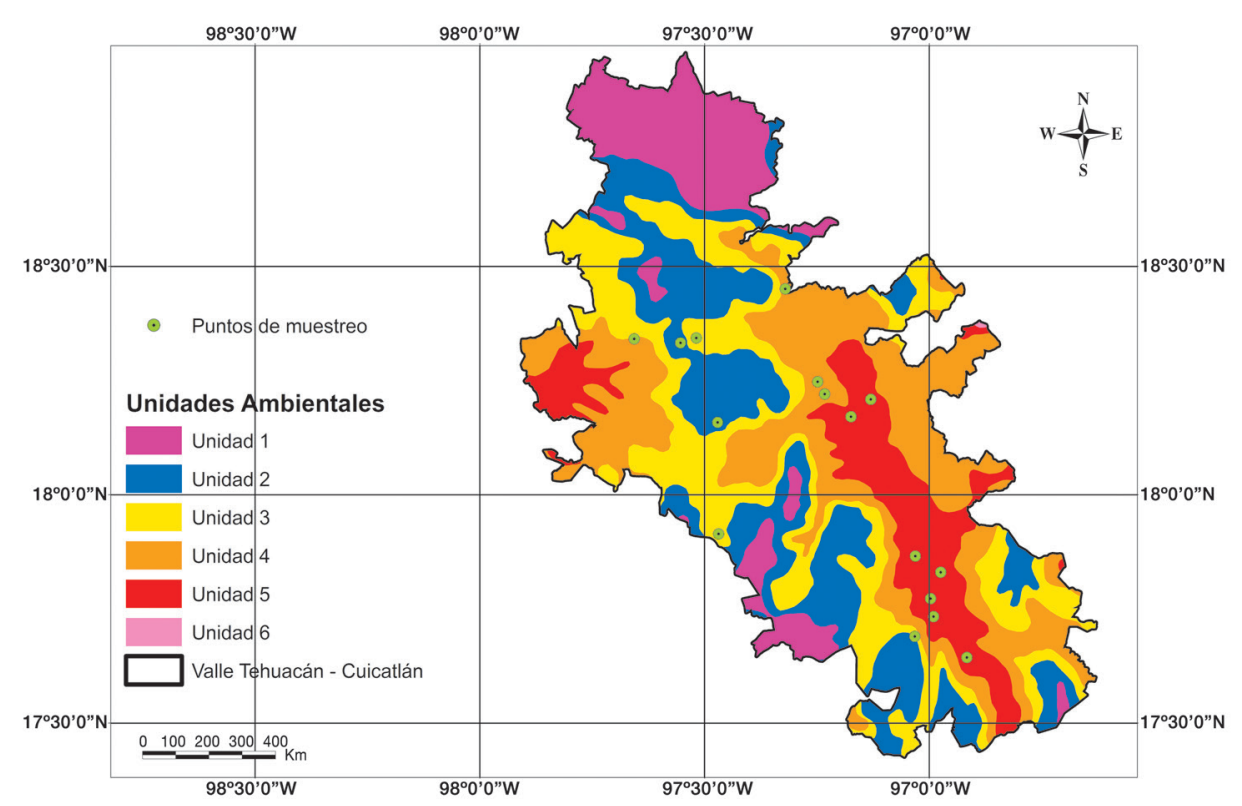

Fig. 1. Unidades Ambientales, sitios (puntos) de muestreo en la Reserva de la Biosfera Tehuacán-Cuicatlán.

Fig. 1. Ecological Land Units, sampling sites (points) in the Tehuacan-Cuicatlan Biosphere Reserve. 
Se destacó la UA3 como la más rica en especies de Jatropha, ya que ahí concurrieron las cinco especies, la UA2 con tres, la UA4 y la UA5 con dos, y en la UA1 y UA6 ninguna. En cuanto a las especies, $J$. neopauciflora y $J$. rzedowskii son las más ubicuas, concurrieron en cuatro UA (2, 3, 4 y 5), J. oaxacana en dos (UA 2 y 3 ), mientras que $J$. rufescens y $J$. ciliata fueron las más restringidas, solo habitaron en la UA3. Esta última especie se encontró en una zona de cultivo de la localidad, alejada de la comunidad vegetal donde se encontró el resto de las especies, por lo que en la figura 1 quedaron representados 16 sitios de muestreo.

Atributos ecológicos: Además de Jatropha, la riqueza de géneros en los puntos de muestreo varió de 16 a 42. San Pedro Jaltepetongo-2 tuvo la mayor riqueza de géneros (42) y especies de Jatropha (3), donde $J$. rzedowskii y $J$. neopauciflora fueron las más abundantes y de mayor cobertura vegetal, comparado con J. oaxacana (Fig. 2).
Los sitios con menor riqueza de géneros (16, 21 y 21) y especies de Jatropha fueron La Mexicana, Santiago Dominguillo y San Nicolás Tepoxtitlán, respectivamente. Las especies de mayor abundancia y cobertura vegetal en todos los sitios fueron $J$. rzedowskii y $J$. neopauciflora.

La vegetación acompañante, más abundante y persistente en el Valle de Tehuacán y Zapotitlán, correspondió con Matorral Xerófito de Agave, Mammillaria, Acacia, Mimosa, Parkinsonia y Baccharis. En la región de la Cañada Oaxaqueña, la vegetación típica de la Selva Baja Caducifolia incluyó abundantes individuos de Bursera, Acacia, Agave, Opuntia y Mimosa.

El Índice de Valor de Importancia (IVI) fue calculado para todos los géneros; sin embargo, se presentó únicamente para Jatropha a nivel de especie (Cuadro 1).

$J$. neopauciflora fue la especie más representada en la zona, mientras que $J$. rufescens sólo se registró en La Mexicana y J.ciliata en

\section{CUADRO 1}

Riqueza genérica e Índice de Valor de Importancia Relativo (IVI\%) por especie de Jatropha

TABLE 1

Richness and Relative Importance Value Index (IVI\%) for genera and Jatropha Species*

\begin{tabular}{|c|c|c|c|c|c|c|}
\hline Localidades & Riqueza de géneros & J.neo & J.rze & J.oax & J.ruf & J.cil \\
\hline Santa Ana Teloxtoc & 29 & 27.20 & 8.56 & $\varnothing$ & $\varnothing$ & $\varnothing$ \\
\hline San Nicolás Tepoxtitlán & 21 & 53.75 & 1.50 & $\varnothing$ & $\varnothing$ & $\varnothing$ \\
\hline Zapotitlán de las Salinas & 29 & 32.50 & 6.00 & $\varnothing$ & $\varnothing$ & $\varnothing$ \\
\hline San Jerónimo Axochitlán & 31 & 7.58 & 7.78 & $\varnothing$ & $\varnothing$ & $\varnothing$ \\
\hline San José Axusco & 24 & 2.04 & 14.8 & $\emptyset$ & $\varnothing$ & $\varnothing$ \\
\hline San Rafael & 36 & 15.26 & 9.92 & $\varnothing$ & $\varnothing$ & $\varnothing$ \\
\hline San Pablo Tepetzingo & 31 & 13.11 & $\varnothing$ & $\varnothing$ & $\varnothing$ & $\varnothing$ \\
\hline Caltepec & 35 & 7.53 & $\varnothing$ & 17.33 & $\varnothing$ & $\varnothing$ \\
\hline San Juan Bautista Cuicatlán & 31 & 25.63 & 3.51 & $\varnothing$ & $\varnothing$ & $\varnothing$ \\
\hline San Gabriel Casa Blanca & 29 & 8.61 & 25.83 & $\varnothing$ & $\varnothing$ & $\varnothing$ \\
\hline Santa María Tecomavaca & 33 & 12.93 & 5.72 & $\varnothing$ & $\varnothing$ & $\varnothing$ \\
\hline Valerio Trujano & 32 & 19.91 & 20.91 & $\varnothing$ & $\varnothing$ & $\varnothing$ \\
\hline Santiago Dominguillo & 21 & 20.58 & $\varnothing$ & $\varnothing$ & $\varnothing$ & $\varnothing$ \\
\hline San Pedro Jaltepetongo-2 & 42 & 5.68 & 6.72 & 2.58 & $\varnothing$ & $\varnothing$ \\
\hline San Pedro Jaltepetongo-1 & $\varnothing$ & $\varnothing$ & $\varnothing$ & $\varnothing$ & $\varnothing$ & $\mathrm{X}$ \\
\hline La Mexicana & 16 & $\varnothing$ & $\varnothing$ & $\varnothing$ & 18.98 & $\varnothing$ \\
\hline
\end{tabular}

$* J . n e o=J$. neopauciflora, J.rze $=J$. rzedowskii, J.oax $=J$. oaxacana, J.ruf $=J$. rufescens, J.cil= J. ciliata .

$\varnothing$ Ausencia de registros de la especie; x Presencia de la especie.

$\varnothing$ Absence of records of the species; $x$ Presence of the species. 

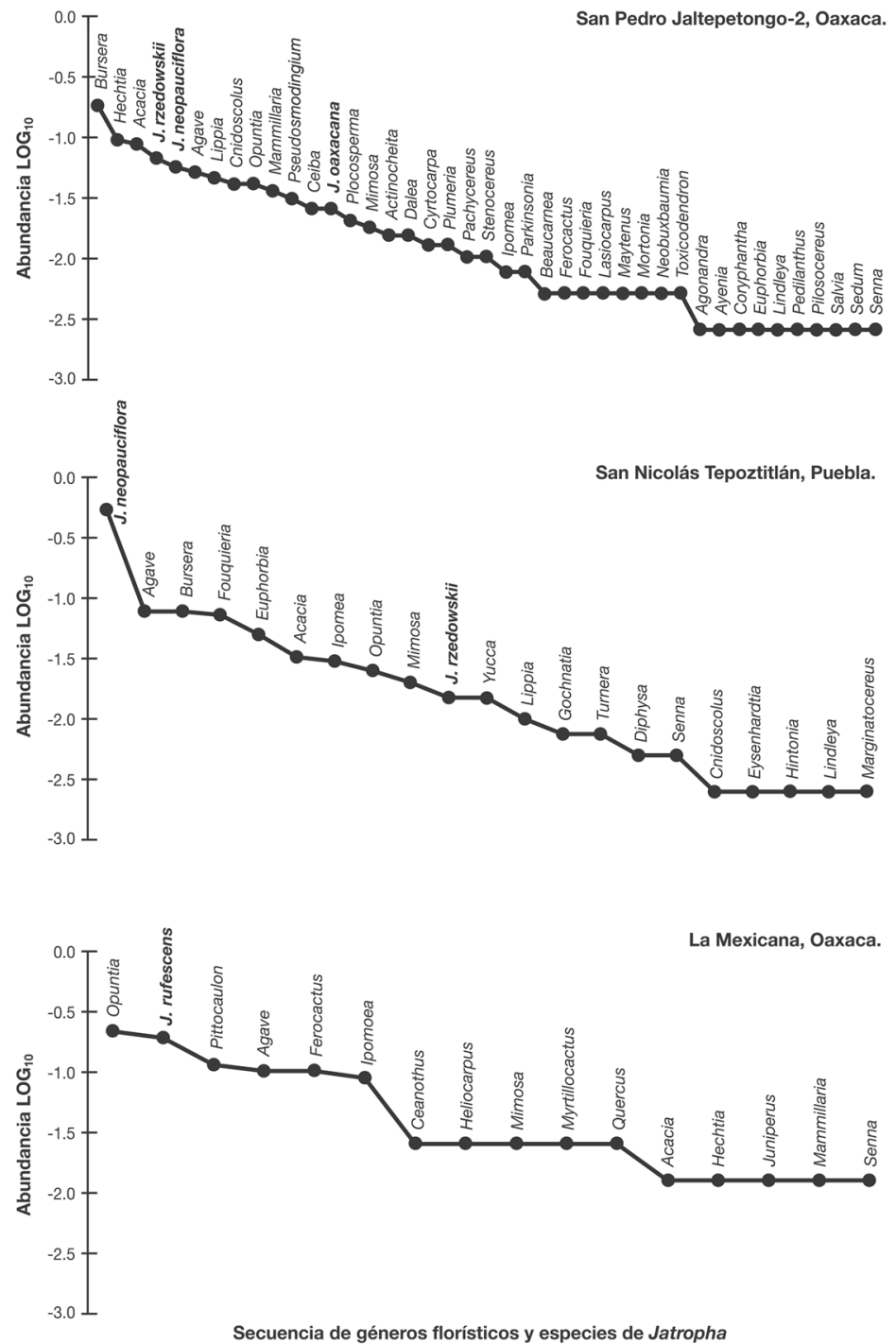

Fig. 2. Curvas de Rango-Abundancia que muestran la comunidad vegetal más rica San Pedro Jaltepetongo-2, Oaxaca, la de riqueza intermedia San Nicolás Tepoztitlán, Puebla y, la comunidad menos rica La Mexicana, Oaxaca.

Fig. 2. Rank-abundance curves illustrating the plant communities with most genera richness San Pedro Jaltepetongo, Oaxaca; medium richness San Nicolas Tepoztitlan, Puebla, and the community lesser richness La Mexicana, Oaxaca.

San Pedro Jaltepetongo-1 a orillas de cultivos dentro de la localidad, por lo cual a esta especie no se le calculó el IVI. El máximo y mínimo IVI fue registrado en San Nicolás Tepoxtitlán para $J$. neopauciflora $(53.75 \%)$ y J. rzedowskii $(1.50 \%)$, respectivamente.

Disturbio: El índice de disturbio fluctuó entre 0.22 y 0.82 , con una media de
0.51. Los sitios más afectados fueron Santiago Dominguillo, Santa Ana Teloxtoc, Zapotitlán de las Salinas y Caltepec, con ID superior a 0.7 (70\%), mientras que los sitios menos afectados fueron San Rafael, San Pedro Jaltepetongo-2 y Santa María Tecomavaca, con ID inferior a 0.3 (30\%). El ACP indicó que los componentes 1 y 2 explican más de $60 \%$ de la variación observada del disturbio total; el primer componente 
con un valor de 6.27 explica $48.27 \%$ de la variación total y el componente 2 con un valor de 2.04 explica $15.74 \%$. En el componente uno, las mayores cargas correspondieron a ganadería, mientras que en el componente dos, correspondieron a la superficie de caminos humanos y extracción de leña. Acorde al ACP de los sitios, por factores de disturbio se destacaron tres grupos, el primero integró a San Gabriel Casa Blanca, San Pedro Jaltepetongo, San Jerónimo Axochitlán y Santa María Tecomavaca, que presentaron el menor índice de disturbio. El grupo dos lo integraron San Pablo Tepetzingo, San José Axusco, La Mexicana, San Juan Bautista Cuicatlán, San Nicolás Tepoxtitlán y Valerio Trujano, con un índice de disturbio medio. En el grupo tres se encontraron a Zapotitlán de las Salinas, Santa Ana Teloxtoc, Caltepec y Santiago Dominguillo, que fueron los sitios con mayor índice de disturbio.

Estado de conservación de las poblaciones de Jatropha: La suma de los valores obtenidos de los cuatro criterios del método de evaluación de riesgo (MER), varió de 0.76 a 2.19 (Cuadro 2). De acuerdo con las categorías de riesgo establecidas por este método, $J$. neopauciflora y J. rzedowskii no se consideraron sujetas a protección; J. oaxacana, mostró necesidad de protección especial y $J$. ciliata y $J$. rufescens se categorizaron en peligro de extinción.

\section{DISCUSIÓN}

Las cinco especies de Jatropha registradas en el presente estudio ya habían sido corroboradas previamente por Hernández-Nicolás y Córdova-Téllez (2012) y coinciden con las especies consignadas por Jiménez (com. pers., 2013) para la región, a excepción de Jatropha andreuxii Müll. Arg., especie no encontrada porque su distribución comienza en la Sierra Mixteca, aledaña a la zona de estudio. Sin embargo, estos resultados contrastan con lo citado por Fresnedo-Ramírez y OrozcoRamírez (2013) y Córdova (com. pers., 2013), quienes para la misma zona estimaron una riqueza de 6 a 11 especies, resultantes de analizar la información de diversos herbarios; la diferencia señalada en el número de especies de Jatropha se atribuye a sinonimias, posibles errores en la identificación del ejemplar y la potencial pérdida, fragmentación o modificación del hábitat, en el que posiblemente existieron esas especies actualmente ausentes, pues en algunos casos la información es de hace más de 50 años; estos diferendos resaltan la importancia de re-inventariar la riqueza de especies y estimar el estado de conservación y riesgo de las especies de Jatropha.

La distribución de las especies de Jatropha en la Reserva de la Biosfera Tehuacán-Cuicatlán se relacionó principalmente con elevación, temperatura y precipitación. Las características

CUADRO 2

Categoría de riesgo para las especies de Jatropha mediante los criterios del MER, NOM-059-ECOL-2010

TABLE 2

Risk categories for Jatropha species using the criteria of the MER, NOM-059-ECOL-2010

\begin{tabular}{lccccc}
\multicolumn{1}{c}{ Criterio } & J. neo & J. rze & J. oax & J. cil & J. ruf \\
Características de la distribución geográfica & 0.36 & 0.45 & 0.73 & 0.82 & 0.82 \\
Características del hábitat & 0.22 & 0.22 & 0.44 & 0.44 & 0.78 \\
Vulnerabilidad biológica intrínseca & 0.17 & 0.17 & 0.30 & 0.43 & 0.39 \\
Impacto de la actividad humana & 0.00 & 0.10 & 0.10 & 0.50 & 0.20 \\
Suma & 0.76 & 0.95 & 1.58 & 2.20 & 2.19 \\
Categoría & Ninguna & Ninguna & Proteción & En Peligro & En Peligro \\
& & & Especial & de Extinción & de Extinción \\
& & & $(P r)$ & & (P)
\end{tabular}


particulares que definieron las unidades ambientales de distribución de Jatropha fueron temperatura mínima superior a $0^{\circ} \mathrm{C}$, precipitación mayor a $200 \mathrm{~mm}$ anuales y elevación inferior a $2500 \mathrm{~m}$; lo que coincide con Govaerts, Frodin, Radcliffe-Smith, y Carter (2010), quienes mencionan que Jatropha se distribuye en regiones cálidas o tropicales secas. Jiménez (com. pers., 2013) identifica a las cinco especies de Jatropha (ciliata, oaxacana, neopauciflora, rzedowskii y rufescens) en las comunidades vegetales de Bosque Tropical Caducifolio, Matorral Xerófilo y Bosque de Pino-Encino, en elevaciones entre los 900 a $2200 \mathrm{~m}$, estas características coinciden con las variables que definen las cuatro UA en donde se encontraron distribuidas las especies de Jatropha.

$J$. ciliata fue registrada a una elevación de $1708 \mathrm{~m}$, clima semicálido, a orillas de cultivos. Rodríguez-Acosta, Vega-Flores, GanteCabrera, y Jiménez-Ramírez (2009), señalaron que tampoco encontraron ejemplares de forma silvestre pero sí herborizados del oriente de Puebla, colectados en Bosque Tropical Caducifolio, entre 1050 y 2 220msnm, cuestión que ratifica Jiménez (com. pers., 2013) al describir que la especie vive asociada a los poblados, además que se ha observado problemas en la posibilidad de cruzamiento, ya que algunos individuos sólo forman flores pistiladas, otros sólo estaminadas, algunos otros ambas, pero no forman semillas, de modo que principalmente se propagan de forma asexual, por lo que se anticipa, que la variación genética y poder de adaptación de $J$. ciliata se estaría vulnerando. Por otro lado, J. rufescens habita de forma silvestre en la ecotonía de Matorral Xerófilo y Bosque Esclerófilo Caducifolio, en lomeríos, cerriles y terrenos escarpados; dicha condición relacionada con el relieve y sequía, posiblemente limitan la amplitud ecológica de su distribución. El grado de disturbio presente en el sitio fue moderado, causado principalmente por la actividad ganadera, extracción de leña y presencia de caminos. J. oaxacana se registró en clima templado con temperatura entre 18 y $22^{\circ} \mathrm{C}$, precipitación anual de 400 y $800 \mathrm{~mm}$, y elevación entre 1500 y $2000 \mathrm{~m}$, en
Bosque Tropical Caducifolio. También existe en Matorral Xerófilo y Bosque de Encino. La cantidad de sitios con estas características en la Reserva fue alta y no concuerda con la escasa distribución, lo que indica que otros factores están influyendo en la presencia-ausencia de la especie. Las especies con mayor distribución en la región de estudio fueron $J$. neopauciflora y $J$. rzedowskii, en hábitats de clima semejantes al caso anterior y mayor amplitud topográfica, desde los $700 \mathrm{~m}$ de altitud, entre el Matorral Xerófilo y el Bosque Tropical Caducifolio. J. neopanciflora por su ubicua presencia, es la única especie del género citada en la guía ecoturística de Tehuacán-Cuicatlán (Téllez et al., 2008).

La mayor riqueza de géneros florísticos y de especies de Jatropha registrados en San Pedro Jaltepetongo fue resultado de la heterogeneidad ambiental y baja intensidad de disturbio. En contraste, la reducida riqueza en Santiago Dominguillo correspondió con el alto grado de disturbio y, en San Nicolás Tepoztitlán, por la errática precipitación. Los géneros que dominaron la comunidad vegetal en el Valle de Tehuacán y Zapotitlán son característicos de Matorral Xerófilo, mientras que en la Cañada Oaxaqueña, correspondieron con los del Bosque Tropical Caducifolio y las tetecheras (Meave, Romero-Romero, Salas-Morales, Pérez-García, \& Gallardo-Cruz, 2012). En Zapotitlán $J$. neopauciflora fue citada como la única especie del género (Arias-Toledo, Valverde-Valdés, \& Reyes-Santiago, 2000), mientras que en este estudio también se registró a J. rzedowskii. Brena (2012), al igual que en el presente estudio, señala la presencia de $J$. neopauciflora y J. rzedowskii en San Rafael, Pue., pero difiere al señalar sólo a $J$. rzedowskii en San Gabriel Casa Blanca, Oax., cuando también existe $J$. neopauciflora en esa localidad. La variación observada entre resultados de los diferentes autores indica la importancia de la contribución del presente trabajo, para el conocimiento de la distribución y riqueza genética de Jatropha en la zona.

En lo referente al IVI, el valor máximo fue obtenido para $J$. neopauciflora de San 
Nicolás Tepoxtitlán, en áreas sobrepastoreadas, condiciones que no favorecen a todas las especies, ya que en este sitio fue registrado el menor IVI para $J$. rzedowskii. También fue importante $J$. rufescens en el único sitio de registro, pero no se puede considerar endémica de la región, como lo señala Méndez-Larios, Ortiz, y Villaseñor (2004), ya que existen otros registros de herbario en Puebla, Jalisco, Colima y Guerrero (Tropicos, 2014), que aún habría que corroborar.

Un caso que debe preocupar es el de $J$. ciliata, especie que florece y no fructifica, lo que anticipa problemas reproductivos, y en consecuencia, reducción de la variación genética, cuestión interesante por resolver desde el punto de vista fitogenético. Es importante destacar que $J$. neopauciflora y $J$. rufescens del estado de Puebla y J. rzedowskii del mismo estado y Oaxaca, son consideradas endémicas para Tehuacán-Cuicatlán (Arias-Toledo et al., 2000; Méndez-Larios et al., 2004). El presente estudio ratificó el endemismo de $J$. neopauciflora y J. rzedowskii pero destacó que tienen amplia distribución en ambos estados. J. rufescens, por los resultados ecológicos se identificó como especie rara, aunque se localiza en otros estados del país, por lo que es necesario verificar la escasez poblacional para sustentar con mayor certeza el estatus de riesgo de la especie. El disturbio representó a sitios de condición regular, medianamente conservados, ya que hubo cargas positivas en varios elementos, entre ellos presencia de ganado y actividades humanas, lo que es acorde con la observación de Martorell y Peters (2005). El estado de conservación de las poblaciones de Jatropha spp. difirió entre especies. Los resultados del MER indicaron que $J$. neopauciflora y $J$. rzedowskii no entran en ninguna categoría de protección especial. No obstante $J$. oaxacana se consideró bajo protección especial $(\mathrm{Pr}), J$. ciliata y $J$. rufescens en peligro de extinción (P), siendo estas últimas las más vulnerables, principalmente por el criterio " $\mathrm{A}$ " que se refiere a la distribución del taxón y el "B" sobre las características del hábitat, pero aún falta mucha información sobre todo en lo concerniente al criterio C, el cual refiere a la vulnerabilidad biológica intrínseca, para ratificar el estatus aquí asignado. Es importante destacar que ninguna de estas especies se encuentra actualmente en la NOM-059-SEMARNAT-2010 (DOF, 2010).

Aunque algunos aspectos del MER son subjetivos, el método facilita la toma de decisiones y permite la generación de hipótesis a probar en futuros estudios (Olson, Lomelí, \& Cacho, 2005). Los resultados aquí descritos sobre la distribución y estado actual de las especies de Jatropha, nos proporcionan el diagnóstico base, para continuar con la elección de estrategias de conservación y la determinación de las especies prioritarias para el Valle de Tehuacán-Cuicatlán, México.

\section{AGRADECIMIENTOS}

Al Sistema Nacional de Recursos Fitogenéticos (SINAREFI-SAGARPA) por el financiamiento otorgado para la investigación. Al personal de la Reserva de la Biosfera Tehuacán-Cuicatlán (CONANP-SEMARNAT) por las facilidades para el estudio de campo. A Alberto Santillán Fernández, por la capacitación para el manejo del software gvSIG. A los habitantes de la región por su participación.

\section{RESUMEN}

Jatropha spp., es un importante recurso fitogenético usado como alimento, medicina y biocombustible. En este estudio verificamos la identidad taxonómica de las especies de Jatropha para la Reserva de la Biosfera TehuacánCuicatlán, y las unidades ambientales (UA) que ocupan. Evaluamos el estado de conservación de sus hábitats y la vulnerabilidad de las poblaciones de Jatropha. Los sitios de muestreo seleccionados en la Reserva fueron 15. El trabajo taxonómico estuvo basado en especímenes, descripciones originales y material tipo de herbarios y aquellos en línea. Las UA fueron clasificadas mediante variables biofísicas y el software gvSIG. Los atributos ecológicos fueron determinados con análisis cuantitativo por el método de cuadrado centrado en un punto; el disturbio fue estimado a través de indicadores de sitio, y el estado de conservación de las poblaciones de Jatropha con el Método para la Evaluación de Riesgo de Extinción de Plantas en México (MER). Jatropha frecuentemente dominó la fisonomía de las comunidades vegetales. La distribución actual de las especies de 
Jatropha en la Reserva estuvo principalmente determinada por las variables altitud, temperatura y precipitación. Las especies confirmadas fueron: Jatropha ciliata Sessé ex Cerv., Jatropha neopauciflora Pax, Jatropha oaxacana J. Jiménez Ram. \& R. Torres, Jatropha rufescens Brandegee, y Jatropha rzedowskii J. Jiménez Ram., las cuales estuvieron distribuidas en cuatro de las seis UA definidas. $J$. neopauciflora y J. rzedowskii son las especies más ampliamente distribuidas; estas últimas especies concurrieron en cuatro, $J$. oaxacana en dos, mientras que $J$. rufescens y $J$. ciliata en una UA, siendo ésta la especie más restringida. La riqueza de géneros en las comunidades asociadas varió entre 16 y 42. Los máximos y mínimos Índices de Valores de Importancia fueron observados en San Nicolás Tepoxtitlan para J. neopauciflora (53.75\%) y J. rzedowskii $(1.50 \%)$. El índice de disturbio varió entre 0.22 y 0.82 , con promedio de 0.51 , mientras que la variable ganado tuvo mayor contribución. Considerando las categorías de riesgo del MER, concluimos que $J$. oaxacana requiere protección especial, mientras que $J$. ciliata y $J$. rufescens fueron especies en riesgo de extinción. El estado de conservación de los hábitats ocupados por poblaciones de Jatropha spp. es moderado, tomando en cuenta la tasa de disturbio. Los principales factores que afectan a los hábitats de Jatropha son el ganado y las actividades humanas; la composición florística de las comunidades vegetales tuvo baja riqueza. Estos resultados apoyan la necesidad de establecer estrategias de conservación para evitar la pérdida de importantes poblaciones silvestres de Jatropha.

Palabras clave: especies de Jatropha, unidades ambientales, Bosque Tropical Caducifolio, disturbio, vulnerabilidad, México.

\section{REFERENCIAS}

Anderson, E. F. (1990). Succulent plant conservation studies and training in Mexico: Stage 1, Part 2, and Stage 2 (Internal report). Washington, D.C., USA.: World Wildlife Fund.

Arias-Toledo, A. A., Valverde-Valdés, M. T., \& ReyesSantiago, J. (2000). Las plantas de la Región de Zapotitlán Salinas, Puebla. D.F., México: Instituto Nacional de Ecología UNAM.

Barredo, C. J. (1996). Sistemas de Información Geográfica y Evaluación Multicriterio. Madrid, España: RA-MA.

Brandegee, T. S. (1910). Plantae Mexicanae Purpusianae. University of California Publications in Botany, 4, 88.

Brena, B. P. (2012). El aprovechamiento y la estructura poblacional de Agave kerchovei Lem., en TehuacánCuicatlán, México (Tesis de Maestría). Colegio de Postgraduados, Texcoco, Estado de México.
Byers, D. S. (1967). The Prehistory of the Tehuacan Valley: Environment and subsistence, edited by D. S. Byers. Austin, Texas, USA.: University of Texas Press.

Cervantes, V. (1794). Discurso pronunciado en el Real Jardín Botánico. Gazeta de Literatura de México, 3(Suppl. 4).

Comisión Nacional para el Conocimiento y Uso de la Biodiversidad (CONABIO). (2013). Valle de Tehuacán-Cuicatlán. Regiones Terrestres Prioritarias de México. Recuperado de http://www.conabio.gob.mx/ conocimiento/regionalizacion/doctos/terrestres.html

Dávila-Aranda, P., \& Lira-Saade, R. (2002). La flora útil de dos comunidades indigenas del Valle de TehuacánCuicatlán: Coxcatlán y Zapotitlán de las Salinas, Puebla (Informe final SNIB-CONABIO proyecto No. T015). D. F., México: Universidad Nacional Autónoma de México. Facultad de Estudios Superiores Iztacala.

Dehgan, B. (2012). Jatropha (Euphorbiaceae), Flora Neotropical Monograph 110. New York, USA.: The New York Botanical Garden Press.

Diario Oficial de la Federación (DOF). (1998). Decreto por el que se declara Área Natural Protegida, con el carácter de Reserva de la Biosfera, a la región denominada Tehuacán-Cuicatlán, ubicada en los estados de Oaxaca y Puebla. Recuperado de http://tehuacancuicatlan.conanp.gob.mx/docs/Tehuacan.pdf

Diario Oficial de la Federación (DOF). (2010). Protección ambiental-Especies nativas de México de flora $y$ fauna silvestres-Categorías de riesgo y especificaciones para su inclusión, exclusión o cambio-Lista de especies en riesgo (NORMA Oficial Mexicana NOM059-SEMARNAT-2010). Recuperado de http://www. profepa.gob.mx/innovaportal/file/435/1/NOM_059_ SEMARNAT_2010.pdf

Feinsinger, P. (2001). Designing field studies for biodiversity conservation. Island, Washington, USA.: The Nature Conservancy.

Fresnedo-Ramírez, J., \& Orozco-Ramírez, Q. (2013). Diversity and distribution of genus Jatropha in Mexico. Genetic Resources and Crop Evolution, 60, 1087-1104.

García, E. (1990). Climas, 1: 4000 000. IV.4.10 (A). Atlas Nacional de México. UNAM, México: Instituto de Geografía.

Govaerts, R., Frodin D. G., Radcliffe-Smith A., \& Carter, S. (2010). World checklist and bibliography of Euphorbiaceae (and Pandaceae). Richmond, Surrey, UK: Royal Botanic Gardens, Kew.

Hernández-Nicolás, N. Y., \& Córdova-Téllez, L. (2012). Diversidad de Jatropha spp. en el Valle de TehuacánCuicatlán. Resumen de Carteles de la Primer Feria de la Agrodiversidad y Agroproductos, SINAREFI, México. Recuperado de http://snics.sagarpa.gob.mx 
Jiménez, R. J. (1985). Dos especies nuevas del género Jatropha en México. Cactáceas y Suculentas Mexicanas, 30(4), 80-84.

Jiménez, R. J., \& Torres C. R. (1994). Especie nueva de la sección Mozinna (Ortega) Pax. Anales Instituto de Biología Universidad Nacional Autónoma de México, Botánica 65(1), 21-29.

Jstor.org (2012). Global Plants (formerly JSTOR Plant Science). Recuperado de http://www.plants.jstor.org

Martínez, G. M., Jiménez, R. J., Cruz, D. R., Juárez, A. E., García, R., Cervantes, A., \& Mejía, H. R. (2002). Los géneros de la familia Euphorbiaceae en México. Anales del Instituto de Biología, UNAM, Serie Botánica, 73(2), 155-281.

Martorell, C., \& Peters E. M. (2005). The measurement of chronic disturbance and its effects on the threatened cactus Mammillaria pectinifera. Biological Conservation, 124, 199-207.

Meave, J. A., Romero-Romero, M. A., Salas-Morales, S. H., Pérez-García, E. A., \& Gallardo-Cruz, J. A. (2012). Diversidad, amenazas y oportunidades para la conservación del bosque tropical caducifolio en el estado de Oaxaca, México. Ecosistemas, 21(1-2), 85-100.

Méndez-Larios, I., Ortiz, E., \& Villaseñor, J. L. (2004). Las Magnoliophyta endémicas de la porción xerofítica de la provincia florística del Valle de TehuacánCuicatlán, México. Anales del Instituto de Biología, Universidad Nacional Autónoma de México, Serie Botánica, 75(1), 87-104.

Mitchell, K. (2007). Quantitative Analysis by the PointCentered Quarter Method. New York, USA.: Department of Mathematics and Computer Science Hobart and William Smith Colleges Geneva, NY. Recuperado de http://arxiv.org/pdf/1010.3303.pdf
Murray, S. (1988). Estadística (2da ed.). Madrid, España: Editorial McGraw Hill.

Olson, M. E., Lomelí, J. A., \& Cacho, N. I. (2005). Extinction threat in the Pedilanthus clade (Euphorbia, Euphorbiaceae), with special reference to the recently rediscovered E. conzattii (P. pulchellus). American Journal of Botany, 92,634-641.

Paredes-Flores, M., Lira, R. S., \& Dávila, P. A. (2007). Estudio etnobotánico de Zapotitlán, Salinas, Puebla. Acta Botánica Mexicana, 79, 13-61. Recuperado de http://www.redalyc.org/articulo.oa?id=57407902

Pax. (1910). Jatropha neopauciflora Pax. Pflanzenr., IV, 147, I (42), 134.

Rodríguez-Acosta, M., Vega-Flores, K., Gante-Cabrera, V. H., \& Jiménez-Ramírez, J. (2009). Distribución del género Jatropha L. (Euphorbiaceae) en el estado de Puebla, México. Polibotánica, 28, 37-48.

Téllez, V. O., Reyes, C. M., Dávila, A. P., Gutiérrez, G. K., Téllez, P. O., Álvarez, E. R., \& Guzmán C. U. (2008). Guía Ecoturística: Las plantas del Valle de Tehuacán-Cuicatlán. México, Facultad de Estudios Superiores Iztacala UNAM. Recuperado de http:// www.poramoralplaneta.com.mx/ganadores/2006/ docs/guia_turistica.pdf

Tropicos.org. (2014). Missouri Botanical Garden. Recuperado de http://www.tropicos.org

Wilson, P. J., \& Provan, J. (2003). Effect of habitat fragmentation on levels and patterns of genetic diversity in natural populations of the peat moss Polytrichum commune. The Royal Society, 270(1517), 881-886.

Zimmerman, C. L., \& Runkle, J. R. (2010). Using ecological land units to conservation planning in a Southwestern Ohio watershed. Natural Areas Journal, 30(1), 27-38. 
